\title{
AN IB-LBM FOR FSI PROBLEMS INVOLVING VISCOELASTIC FLUIDS AND COMPLEX GEOMETRIES
}

\author{
Jingtao MA ${ }^{1}$, Zhen Wang ${ }^{2}$, Yi Sui ${ }^{2}$, John Young ${ }^{1}$, Joseph C.S. Lai ${ }^{1}$ and Fang-Bao Tian ${ }^{1}$ \\ ${ }^{1}$ School of Engineering and Information Technology, University of New South Wales, Canberra, ACT \\ 2600, Australia \\ ${ }^{2}$ School of Engineering and Materials Science, Queen Mary University of London, London E1 4NS, \\ UK
}

Key words: Immersed-boundary method, lattice Boltzmann method, fluid-structure interaction, viscoelastic fluids

\begin{abstract}
An immersed boundary-lattice Boltzmann method (IB-LBM) for fluid-structure interaction (FSI) problems involving viscoelastic fluids and complex geometries with a few validation cases is presented in this paper. In this method, the lattice Boltzmann method is used to solve the fluid dynamics and the constitutive equations of viscoelastic fluids. An artificial damping is introduced to enhance numerical stability in solving the constitutive equations. A hybrid of the finite difference method (2D and 3D rigid particles) and the finite element method (3D capsule) is employed to solve the structural dynamics. The interaction between the solid structure and the fluid is achieved by an immersed boundary method. The present method and models are validated by several cases including 2D Oldroyd-B channel flow, 2D lid-driven cavity flow, 2D Oldroyd-B flow over a confined cylinder, a 2D rigid particle migration in an Oldryod-B Couette flow, a spherical particle rotation in an Oldroyd-B shear flow, a spherical particle settling in a Newtonian fluid, and the deformation of a spherical capsule in a long channel filled with a Newtonian fluid.
\end{abstract}

\section{Introduction}

Non-Newtonian fluids can be involved in many industrial and biological systems, and typical examples of non-Newtonian fluids are polymer solutions, shampoo, paints, food and mucus layer. In many scenarios, the interaction between non-Newtonian fluids and solid structures can be found. For example, a free swimmer (e.g., microorganism or sperm) may encounter complex fluids (non-Newotnian fluids, e.g., substrates or cervical fluid) $[1,2,3,4,5]$. Sometimes, it is difficult to conduct the experimental study on fluid-structure interaction (FSI) problems due to the small scale of the structure (e.g., microorganisms in the order of $\mu m$ ). In this case, the numerical method can be a suitable tool to investigate these problems.

Many previous numerical investigations on FSI problems involving non-Newtonian fluids have been based on body-conformal grids, and methods of these investigations normally include the arbitrary Lagrangian Eulerian-finite element method (ALE-FEM) [6,7] and the deforming-spatial-domain/stabilized space-time (DSD/SST) method $[8,9,10]$. The disadvantage of these methods is obvious: the mesh needs to be regenerated to ensure the quality of mesh when large deformation/displacement is involved [11, 12, 13]. In contrast, IB-LBM (based on the non-conformal mesh) provides a convenient alternative for 
FSI problems involving moving boundaries and large deformation/displacement. Recently, IB-LBM has been used to study FSI problems involving viscoelastic fluids $[14,15,16]$. Specifically, a numerical study on settling of rigid particles in viscoelastic fluids was conducted by Goyal and Derksen [14]. In this research, the lattice Boltzmann method (LBM) was used to solve the flow of fluids, the finite volume method (FVM) was utilized to solve the constitutive equations of viscoelastic fluids, and the immersed boundary method (IBM) was utilized to achieve the interaction between fluids and rigid particles. In addition, the accuracy of this method was confirmed by different validation cases (2D Oldroyd-B flow over a cylinder, a 3D spherical particle rotation in an Oldroyd-B shear flow, and a 3D spherical particle sedimentation in Newtonian fluid under gravity). More recently, LBM and IBM were combined to study the motion of a massless flexible sheet in viscoelastic flows by Zhu [15, 17], where the LBM originally proposed by Malaspinas et al. [18] was utilized to solve the viscoelastic flow, and the FSI was enforced by IBM. In this study, it was reported that the motion of the sheet may be hindered with the inclusion of the fluid viscoelasticity.

Despite the studies mentioned above, more efforts are still necessary to extend IB-LBM to FSI problems in viscoelastic flows involving large deformation and complex geometries. In this work, an IB-LBM for FSI problems involving viscoelastic fluids and complex geometries is presented. The details of this method were introduced in our recent work [16]. Here we are going to briefly introduce this method with a few new validation cases. In this method, the LBM proposed by Malaspinas et al. [18] is used to solve the Navier-Stokes equations and the constitutive equations of the viscoelastic model, the finite difference method (FDM) and the finite element method (FEM) are employed for the dynamics of solid structure (FDM for 2D and 3D rigid particles, and FEM for 3D capsule), and the FSI is accomplished with the IBM.

2 Mathematical model and numerical method The dynamics of an incompressible fluid are described.
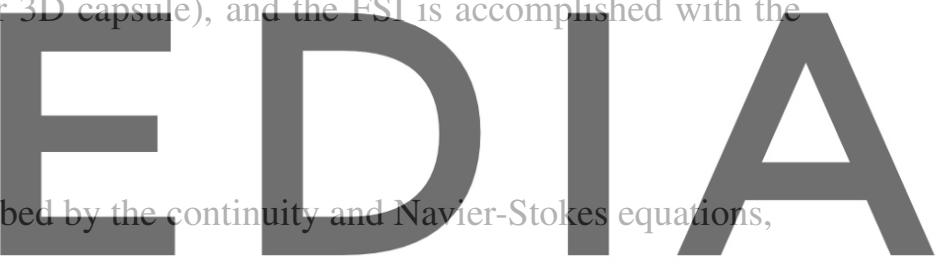

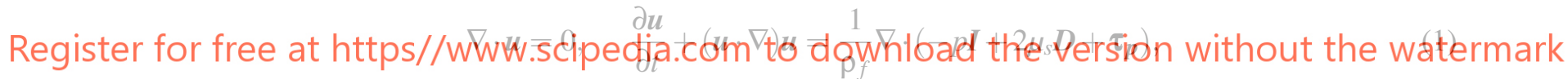

where $\rho_{f}$ is the fluid density, $u$ is the fluid velocity, $p$ is the pressure, $\mu_{s}$ is the solvent dynamic viscosity, $\boldsymbol{I}$ is the identity tensor, $\boldsymbol{D}=\frac{1}{2}\left(\nabla \boldsymbol{u}+(\nabla \boldsymbol{u})^{T}\right)$ is the strain rate tensor, and $\boldsymbol{\tau}_{\boldsymbol{p}}$ is the viscoelastic stress tensor which accounts for the effect of the polymers on the solvent. In this work, one of the most commonly used model (Oldroyd-B model) is used to determine $\tau_{p}[14,18,19]$. In this model, the viscoelastic stress tensor $\tau_{p}$ can be described by the conformation tensor $\boldsymbol{C}$ (a statistical indicator of the orientation of the polymer molecules) $[14,18]$,

$$
\tau_{p}=\frac{\mu_{p}}{\lambda}(\boldsymbol{C}-\boldsymbol{I}) \quad(\text { Oldroyd }-\mathrm{B}),
$$

where $\mu_{p}$ and $\lambda$ are respectively the dynamic viscosity and relaxation time of the polymer. The conformation tensor $\boldsymbol{C}$ is determined by the following transport equations,

$$
\frac{\partial \boldsymbol{C}}{\partial t}+(\boldsymbol{u} \cdot \nabla) \boldsymbol{C}=-\frac{1}{\lambda}(\boldsymbol{C}-\boldsymbol{I})+\boldsymbol{C} \cdot \nabla \boldsymbol{u}+(\nabla \boldsymbol{u})^{T} \cdot \boldsymbol{C} \quad(\text { Oldroyd }-\mathrm{B}) .
$$


The motion of the rigid particle is governed by the Newton's second law,

$$
m_{p} \frac{d \boldsymbol{V}_{p}}{d t}=\boldsymbol{F}_{e x t}, \quad I_{p} \frac{d \boldsymbol{\Omega}_{p}}{d t}=\boldsymbol{T}_{e x t}
$$

where $m_{p}$ and $I_{p}$ respectively denote the mass and moment of inertia of the particle, $V_{p}$ and $\Omega_{p}$ are respectively the translational and rotational velocity of the particle, and $\boldsymbol{F}_{e x t}$ and $\boldsymbol{T}_{\text {ext }}$ are respectively the external force and moment exerted on the particle.

The membrane of the 3D capsule is treated as a zero-thickness elastic surface. Here, the 3D capsule model is introduced from an energy point of view since it is very difficult to describe the dynamics of a 3D capsule in a Cartesian system. The deformation of the capsule may induce stretching and bending energies. Here, we employ the Skalak's law to compute the stretching force to be consistent with the previous investigation [20,21]. In this model, the strain energy function due to the stretching deformation is given by

$$
W^{S}=\int_{A}\left[\frac{E_{s}}{12}\left(I_{1}^{2}+2 I_{1}-2 I_{2}\right)+\frac{E_{a}}{12} I_{2}^{2}\right] d A
$$

where $W^{S}$ is the total strain energy density, $E_{s}$ is the surface elasticity modulus, and $E_{a}$ is elastic modulus for area dilation for surface-area conservation. The membrane bending resistance is described by the Helfrich bending energy [22, 23],
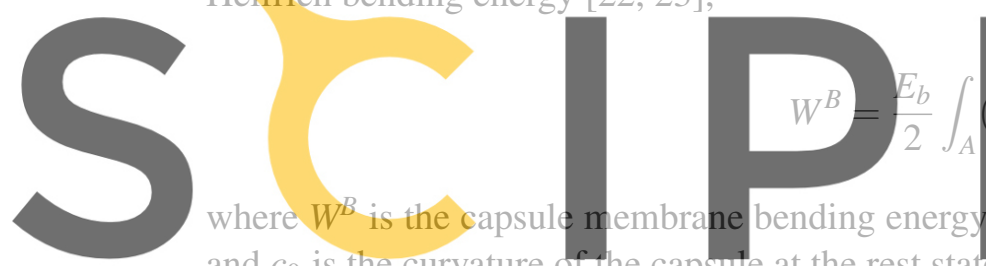
and $c_{0}$ is the curvature of the capsute at the rest stat
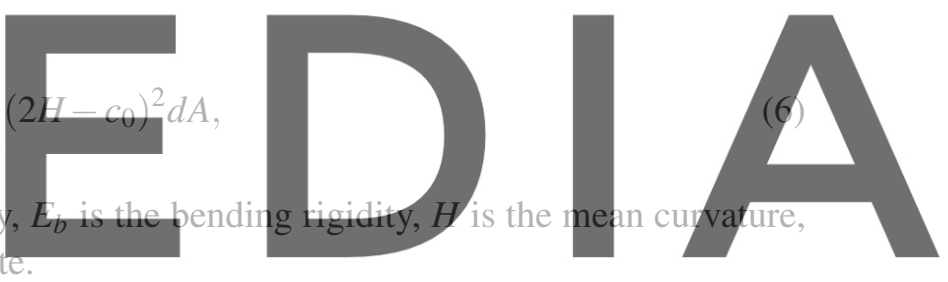

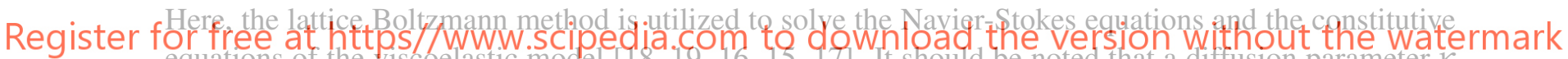
equations of the viscoelastic model $[18,19,16,15,17]$. It should be noted that a diffusion parameter $k$ is normally involved in this method (in the transport equations of the conformation tensor $C$ ) to improve the stability of simulations, and more details can be found in Refs. $[18,16]$. The structure dynamics including 3D capsule and 2D and 3D rigid particles are solved by the finite difference method (2D and 3D rigid particles) and the finite element method (3D capsule) [16]. Here, the finite difference method is employed for rigid particles due to its convenience and simplicity, and the finite element method is used for 3D capsule due to its more straight forward computation of the stretching and bending forces on 3D deformable structures. The interaction between the fluid and the solid structure is achieved by using the immersed boundary method $[16,11,24]$. The immersed boundary-lattice Boltzmann method used here has been extensively validated in different flows in our previous work [16], and details of this method and validation cases as well as its applications can be found in our previous studies [16, 11, 25, $26,27,28]$. Here, this method is further validated by several cases: 2D Oldroyd-B channel flow, 2D lid-driven Oldroyd-B cavity flow, 2D Oldroyd-B flow over a confined cylinder, a 2D neutrally buoyant particle migration in an Oldroyd-B Couette flow, a 3D neutrally buoyant spherical particle rotation in an Oldroyd-B shear flow, a spherical particle settling under gravity in a Newtonian fluid, and a 3D spherical capsule deformation in a channel with a the square cross-section. 
(a)

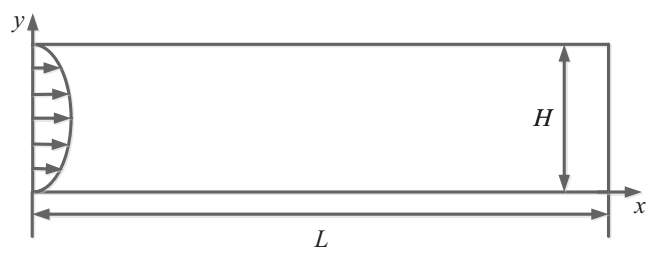

(b)

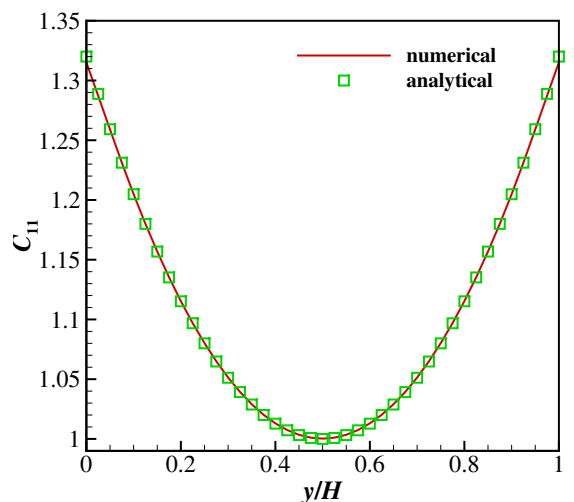

Figure 1: 2D Oldroyd-B channel flow: (a) The schematic diagram for the 2D Oldroyd-B flow in a long channel. (b) Comparison of analytical and numerical solutions for the $C_{11}$ component of the conformation tension at the outflow boundary.

\section{Validation}

\subsection{D Oldiroyd-B channel flow}

Here, the 2D flow of an Oldroyd-B fluid in a long channel is conducted to validate the solver of the viscoelastic fluid. The computational domain is $[0, L] \times[0, H]$ with $L=8 H$ (as shown in Fig. 1(a)).

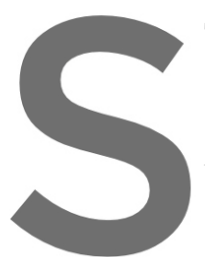
The velocity profile predicted by $u_{y}=0$ is applied at the inl stationary walls, and Newmann bourl boundary. The non-dinensional parameters gove $\rho_{f} U_{c} H / \mu_{0}$, the Weissenberg number $W i=\lambda U_{c} / H$,
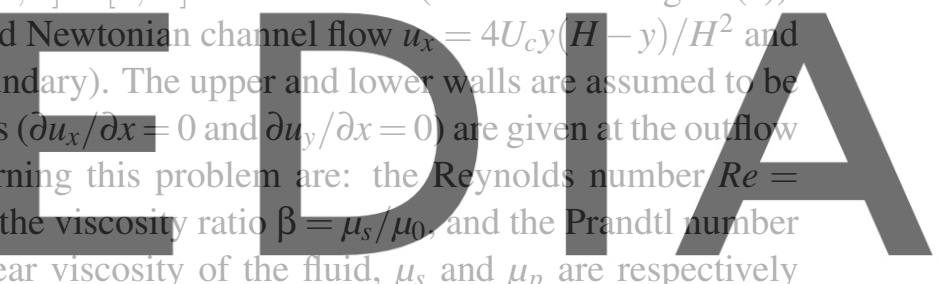

$\operatorname{Pr}=\kappa /\left(\dot{\gamma} H^{2}\right)$, where $\mu_{0} \equiv \mu_{s}+\mu_{p}$ is the zero-shear viscosity of the fluid, $\mu_{s}$ and $\mu_{p}$ are respectively the viscosities of the solvent and the polymer, and $\dot{\gamma}=2 U_{c} / H$ is wall the shear rate. Here $R e=0.05$, Register far free at htgtps/duw wrw.scipedia.com to download the version without the watermark

The grid spacing used in the simulations is $h=\Delta x=\Delta y=0.025 \mathrm{H}$. Fig. 1(b) shows that the numerical solution for $C_{11}$ component of the conformation tension $C$ of the fully developed state at the outflow boundary, and the numerical solution agrees well with the analytical solution $\left(C_{11}=2 \lambda^{2}\left(\partial u_{x} / \partial y\right)^{2}\right)$.

\subsection{D lid-driven Oldroyd-B cavity flow}

The 2D lid-driven Oldroyd-B cavity flow is performed to further validate the solver of the viscoelastic fluid. The definition of the computational domain, the associated coordinate system and the boundary conditions are shown in Fig. 2. The length of the edge of the cavity is $L$. A velocity of $U(x)=$ $8 U_{c}\left(\frac{x}{L}\right)^{2}\left(1-\frac{x}{L}\right)^{2}(1+\tanh (8 t-4))$ [29] is applied to the upper boundary of the cavity. The non-dimensional parameters of this problem are: the Reynolds number $R e=\rho_{f} U_{c} L / \mu_{0}$, the Weissenberg number $W i=$ $\lambda U_{c} / H$, the viscosity ratio $\beta=\mu_{s} / \mu_{0}$, and the Prandtl number $\operatorname{Pr}=\kappa /\left(\dot{\gamma} L^{2}\right)$ with $\dot{\gamma}=U_{c} / L$. Here, $R e=1.0, W i=1.0, \beta=0.5$, and $\operatorname{Pr}=1 \times 10^{-4}$.

Two different grid spacings $h=\Delta x=\Delta y=0.005 \mathrm{~L}$ and $0.0025 \mathrm{~L}$ are used here to verify the mesh size independence of the solver. Fig. 3 shows the profiles of $u_{x}$ along the vertical centerline and $u_{y}$ along 


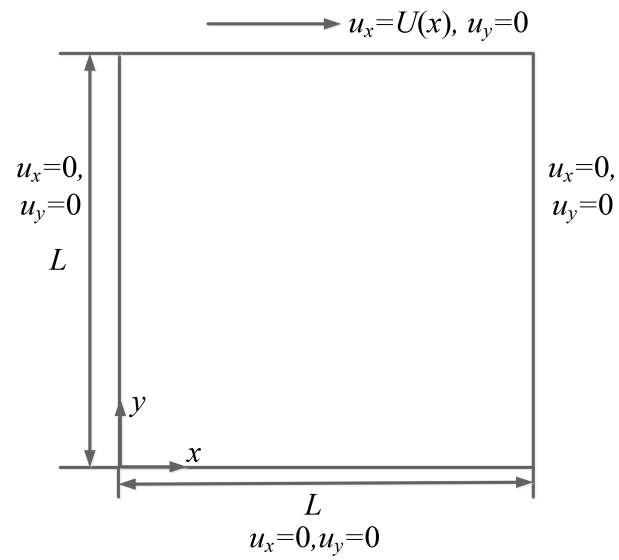

Figure 2: The schematic diagram for the 2D lid-driven Oldroyd-B cavity flow.
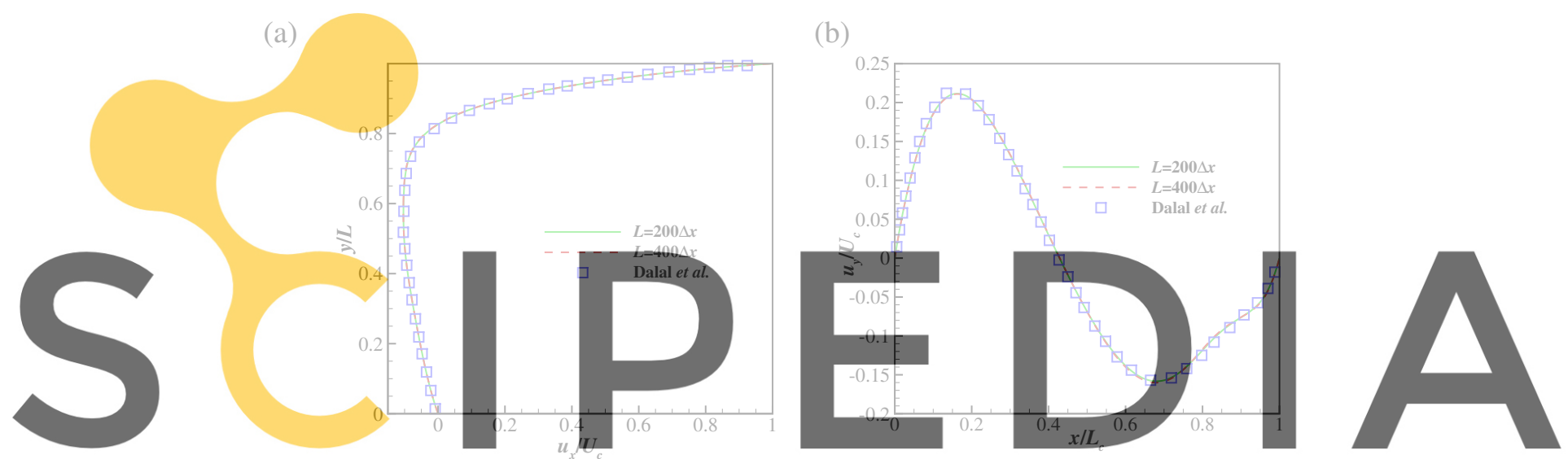

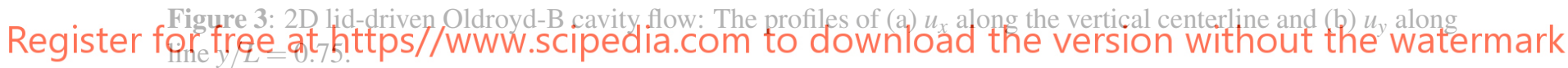

the line $y / L=0.75$ for different mesh sizes. It is observed that the profiles are mesh independent. In addition, the results agree with those obtained by Dalal et al. [29] based on the finite difference method (FDM) which normally requires stabilization strategies for high Weissenberg number problems.

\subsection{D Oldroyd-B flow over a confined cylinder}

Here, the 2D flow of an Oldoryd-B fluid over a confined cylinder is performed. The schematic diagram for this problem is shown in Fig. 4(a). The computational domain used is $[0, L] \times[0, H]$ with $L=30 R$ and $H=4 R$ ( $R$ is the radius of the cylinder). The center of the particle is initially placed at $(10 R, 0)$. The velocity profile predicted by the fully developed Newtonian channel flow $u_{x}=6 U_{c} y(H-y) / H^{2}$ and $u_{y}=0$ is applied at the inlet of the channel (left boundary). The upper and lower walls are assumed to be stationary walls, and Newmann boundary conditions $\left(\partial u_{x} / \partial x=0\right.$ and $\left.\partial u_{y} / \partial x=0\right)$ are given at the outflow boundary. The non-dimensional parameters governing this problem are: the Reynolds number $R e=\rho_{f} U_{c} R / \mu_{0}$, the Weissenberg number $W i=\lambda U_{c} / R$, the viscosity ratio $\beta=\mu_{s} / \mu_{0}$, and the Prandtl 
(a)

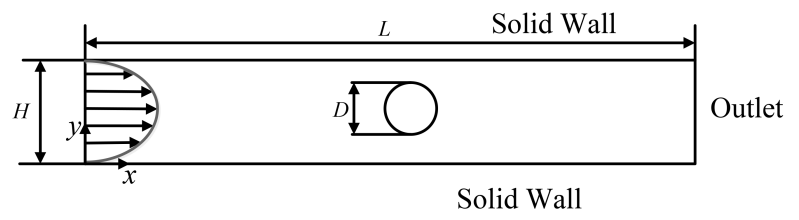

(b)

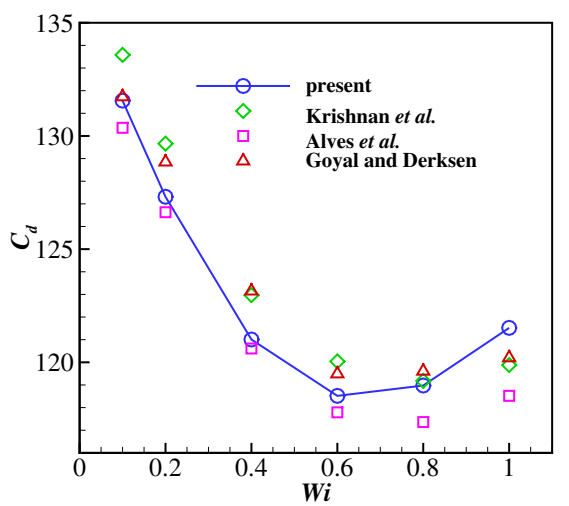

Figure 4: 2D Oldroyd-B flow over a confined cylinder: (a) The schematic diagram for the 2D Oldroyd-B flow over a confined cylinder. (b) The drag coefficient $\left(C_{d}\right)$ as a function of Weissenberg number $(W i)$.

number $\operatorname{Pr}=\kappa /\left(\dot{\gamma} R^{2}\right)$, where $\dot{\gamma}=3 U_{c} / H$ is the shear rate. Here, $R e=0.05, \beta=0.59, \operatorname{Pr}=6.67 \times 10^{-3}$, and $W i=0,0.1,0.2,0.4,0.6,0.8$ and 1.0 .

The grid spacing used in the simulations is $h=\Delta x=\Delta y=0.02 R$. Fig. 4(b) shows the drag coefficient $\left(C_{d}=\frac{F_{d}}{\mu_{0} U_{c}}\right.$ where $F_{d}$ is the drag force exerted on the cylinder by the ambient fluid) as a function of the Weissenberg number $W i$, and the present results agree well with the results in Refs. [30, 31, 14].

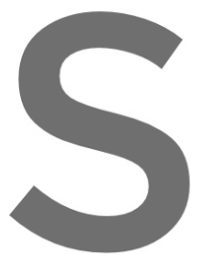

3.4 A 2 D neutrally buoyant particie mighation in an Oldioyd-B Couretteflow
The dynamics of a 2D neutrally buoyant particle nigration in an Oldroyd-B fluid Couette flow is con-
sidered here to validate the 2D FSI procedure. The definition of the physical problem and the associated
coordinate system is shown in Fig. $5(\mathrm{a})$. The radius of the circular particle is $R$, and the compulational
domain spans from $(-5 R, 0)$ to $(5 R, 8 R)$, respectively. Periodic boundary condition is applied at the inlet and outlet. Constant velocity $U_{G}$ is imposed at the bottom boundary, while $-U_{G}$ is applied at the

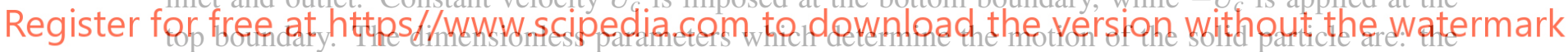

Reynolds number $R e=\rho_{f} U_{c} R / \mu_{0}$, the Weissenberg number $W i=U_{c} \lambda / R$, the viscosity ratio $\beta=\mu_{s} / \mu_{0}$ and the Prandit number $\operatorname{Pr}=\kappa / \gamma(2 R)^{2}$. Here $R e=5, w i=1.0$, and $\beta=1 / 8$.

The grid spacing is $h=\Delta x=\Delta y=0.025 R$. To determine the suitable value of the diffusive constant $\kappa$ used in this problem, four different values of $\operatorname{Pr}$ are used in the simulations: $1.25 \times 10^{-4}, 1.25 \times 10^{-3}$, $1.25 \times 10^{-2}$ and $5 \times 10^{-2}$. Fig. 5(b) shows the time history of the particle center in $y$ direction for initial position of $y_{0}=0.25 \mathrm{~W}$ and different values of $\mathrm{Pr}$. From Fig. 5(b), it is found that the velocity of lateral migration of the particle decreases with the increase of $\mathrm{Pr}$. In addition, the particle migration is significantly affected by the artificial viscosity introduced when $\operatorname{Pr}$ is in the order of $10^{-2}$. Therefore, in order to avoid the effect of the artificial viscosity, the Prandtl number $\operatorname{Pr}$ should be less than $1 \times 10^{-2}$ (preferable order is $10^{-4}$ ). The comparison between present results and the previous results obtained by employing arbitrary Lagrangian-Eulerian finite element method (ALE-FEM) from Huang et al. [32] and Lv et al. [33] in Fig. 5(b) shows good agreement. The ALE-FEM used by previous studies [32, 33] normally requires the regeneration of the mesh when large deformation is involved (as stated in Section 1), which the present method does not require, hence reducing the computational cost. 
(a)

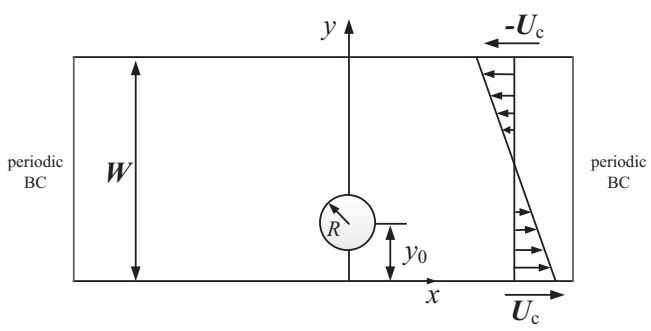

(b)

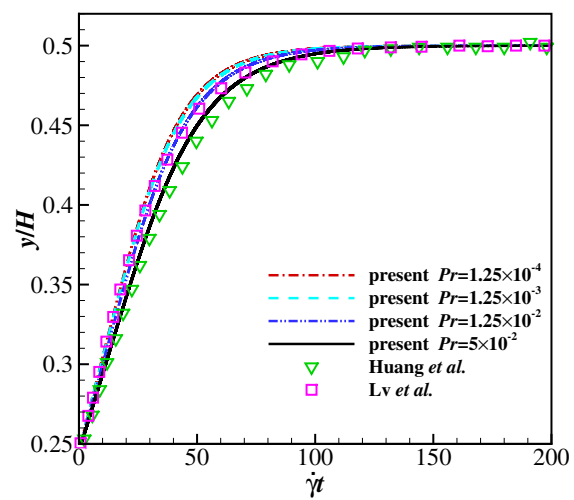

Figure 5: A 2D neutrally buoyant particle migration in an Oldroyd-B Couette flow: (a) Schematic illustration of a $2 \mathrm{D}$ buoyant particle migration in an Oldroyd-B Couette flow. (b) The lateral migration of the particle at initial position $y_{0}=0.25 \mathrm{~W}$ and different values of the Prandtl number $\mathrm{Pr}$.

\subsection{A 3D neutrally buoyant spherical particle rotation in an Oldroyd-B shear flow}

Here, we consider the rotation of a neutrally buoyant spherical particle in an Oldroyd-B shear flow to further validate the present method. As shown in Fig. 6, the simulation is conducted in a cuboid domain of $[-8 R, 8 R] \times[-4 R, 4 R] \times[-4 R, 4 R]$ and the center of the particle is initially placed at $(0,0,0)$.

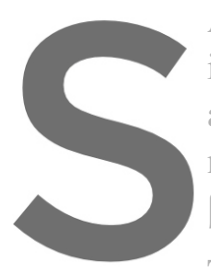
A simple shear flow is in opposite directions in applied at the other four number $R e=\rho_{f} \dot{\gamma}(2 R)^{2}$ $\beta=\mu_{s} / \mu_{0}$, and the Pranc
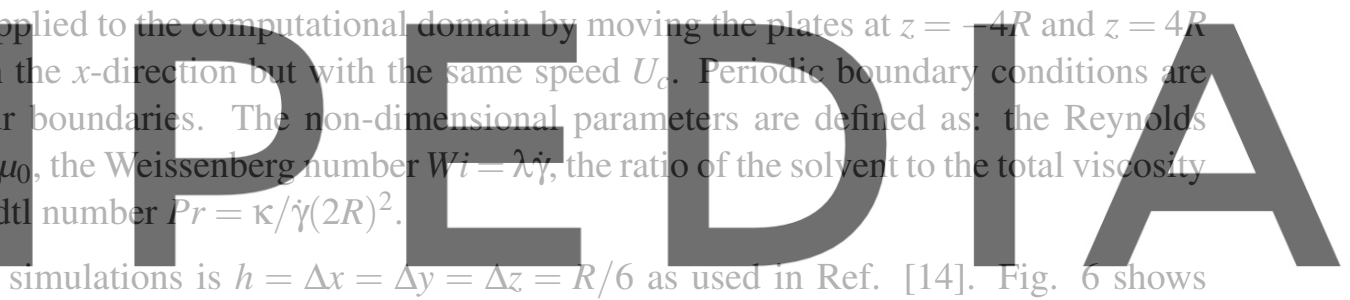

The grid spacing in the simulations is $h=\Delta x=\Delta y=\Delta z=\pi / 6$ as used in Ref. $[14$. $R=0.5$. It is

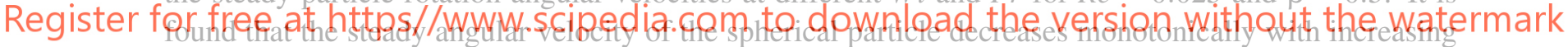

Wi which means the viscoelasticity of the fluid hinders the rotation of the particle in the Oldroyd-B shear flow. The angular velocities of the particie here show good agreement with previous numerical results by Snijkers et al. [34] using FEM-ALE and Goyal and Derksen [14] using an the immersed boundary-lattice Boltzmann-finite volume method (IB-LBM-FVM) demonstrating the accuracy of the present method to simulate 3D particle motion in viscoelastic fluids. It is also found that when $\operatorname{Pr} W i \leq 0.001$, decreasing $\operatorname{PrWi}$ does not have a significant influence on the angular velocity of the particle. This means the damping effects on the simulations are insignificant compared with the relaxation effects when the product $\mathrm{PrWi}$ is in the order of $\mathrm{O}\left(10^{-3}\right)$ or less.

\subsection{A spherical particle settling under gravity in a Newtonian fluid}

A spherical particle with diameter $d$ settling under gravity in a square channel filled with a Newtonian fluid is presented in this section to further validate the 3D rigid particle model. Miyamura et al. [35] experimentally investigated the effects of the wall on the the spherical particle settling in square cylinders, and suggested that the terminal settling velocity of the particle is significantly influenced by the wall. Fig. 


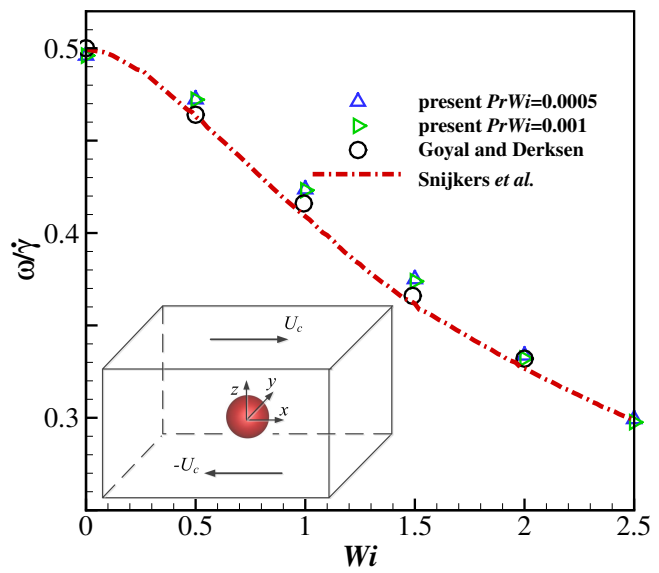

Figure 6: A spherical particle rotation in an Oldroyd-B shear flow: The effect of $W i$ on the particle steady rotation angular velocity. Inset: schematic illustration of a spherical particle rotation in an Oldroyd-B shear flow.

7 (a) shows the physical problem and the coordinate definition of the present simulation. The computation domain is $[0, L] \times[0, L] \times[0, H]$, and the gravity is applied in the negative $z$ direction. The particle is initially released at the center of the cross-section of the channel and $z=0.8 H(H=5 L)$ with zero velocity. The non-dimensional parameters are defined as: the Reynolds number $R e=\frac{\rho_{f} U_{t} d}{\mu}=0.36$, the

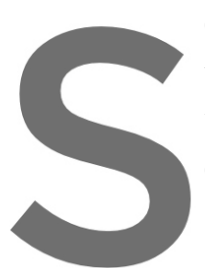
density ratio $\frac{\rho_{p}}{\rho_{f}}=2.5$

the unconfined terminal velocity $U_{t}$ is prescribe drag force and buoyancy

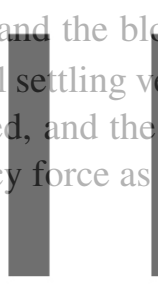

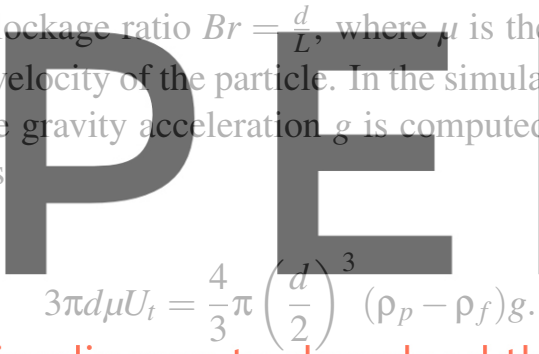

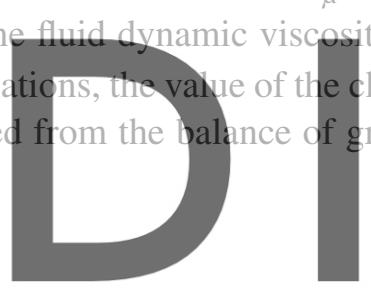

$3 \pi d \mu U_{t}=\frac{4}{3} \pi\left(\frac{d}{2}\right)$

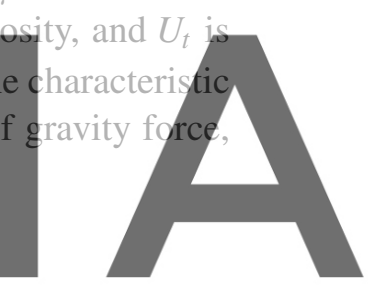

(7)

Register for free at https//www.scipedia.com to download the version without the watermark The wall effect is quantified by the non-dimensional terminal velocity $\zeta=U_{0} / U_{t}$, where $U_{0}$ is the particle terminal settling velocity with the nearby boundary walls. Fig. 7(b) shows the wall effects on the particle terminal settling velocity at different spatial resolutions and characteristic velocities, and the comparison of results from present simulations with the best curve fit to experimental data of Miyamura et al. [35] and numerical results from Aidun et al. [36]. It is observed that the non-dimensional terminal settling velocities of the particle $U_{0} / U_{t}$ declines with the decrease of the characteristic velocity for a specific blockage ratio, and reaches a convergency at $U_{t}=0.0005$ (in LBM unit). Here $U_{t}$ is used to test the effects of the dimensionless $\Delta t$ and $\tau$ on the results. In addition, with the increase of the spatial resolution, the non-dimensional particle terminal settling velocity approaches the experimental results, and present results show good agreement with the results from previous experimental and numerical investigations.

\subsubsection{A 3D spherical capsule deformation in a channel with a the square cross-section}

The deformation of a 3D spherical capsule in a channel (filled with a Newtonian fluid) with a square cross-section is considered here to validate the $3 \mathrm{D}$ capsule model. The simulation is conducted in a cuboid box as shown in Fig. 8(a). The computational domain is $[0, L] \times[0, H] \times[0, H]$ with $L=10 H$. 
(a)

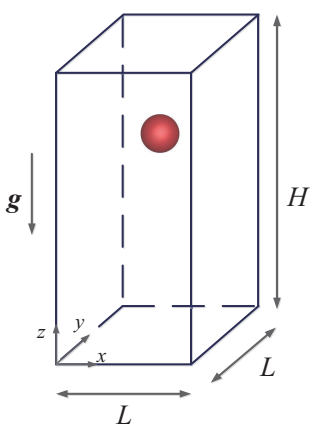

(b)

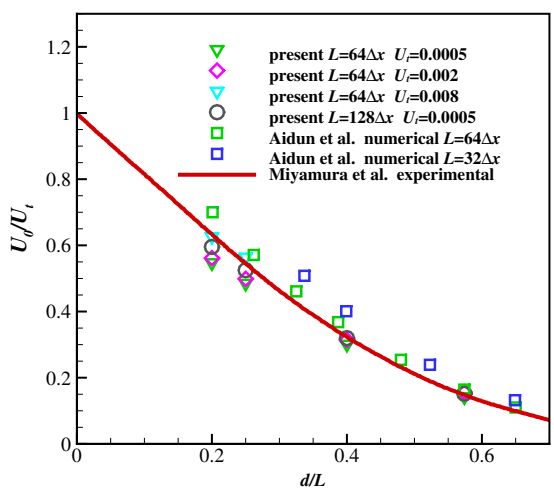

Figure 7: A spherical particle settling under gravity in a Newtonian fluid: (a) Schematic illustration of a spherical capsule settling in a square channel filled with Newtonian fluid. (b) The particle terminal settling velocity under different blockage ratios.

The capsule has a radius of $R$, and the elastic force of the capsule is governed by the SK model with $C=1$ [16]. The fully developed Poiseuille flow is applied to the inlet of the channel (i.e., the left boundary), and the axis velocity distribution $\boldsymbol{u}=u(y, z) \boldsymbol{e}_{x}$ is given by [37]
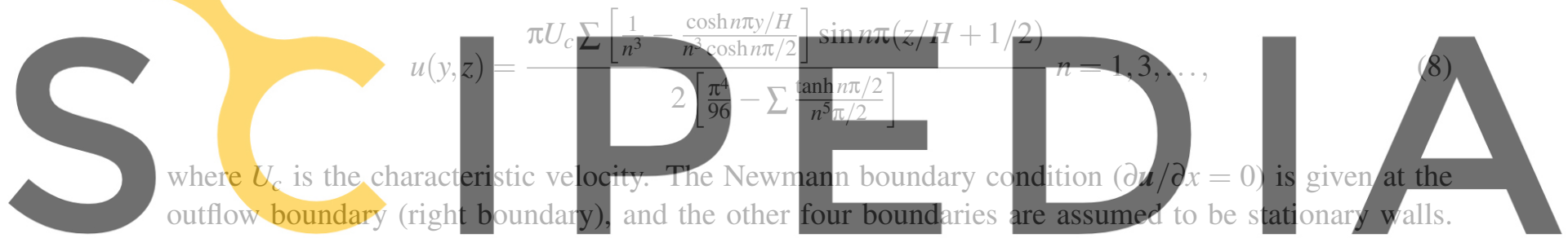

outflow boundary (right boundary), and the othe

The non-dimensional parameters are defined as: the Reynolds number $R e=\rho_{f} U_{c} H / \mu_{S}$, the Capillary

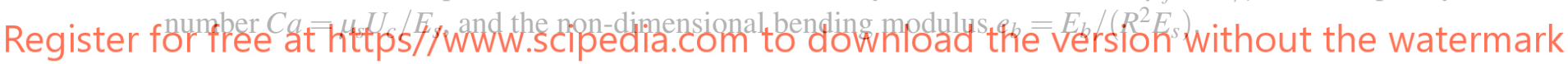

Following the study by Wang et al. [20], the grid spacing is $h=\Delta x=\Delta y=\Delta z=0.02 H, R e=0.25$,

$e_{b}=0,2 R=0.85 H$ and $C a=0.3$. As shown in Fig. 8(b), the equilibrium profiles of the capsule when discretized into 5120 and 20480 elements are in very good agreement with the numerical results of Wang et al. [20] (32768 elements), but they are slightly different from the results by Hu et al. [21]. This is because the Reynolds number used in the simulations by Wang et al. [20]. The Reynolds number used in the present study and Wang et al. [20] is 0.25 , but very small (approximately 0) in Hu et al. [21].

\section{CONCLUSIONS}

A numerical method for FSI problems involving viscoelastic fluids and complex geometry is presented in this work. In this method, the lattice Boltzmann method is used to solve the fluid dynamics and the constitutive equations of viscoelastic fluids. The interaction between the solid structure and the fluid is achieved by employing the immersed boundary method. The finite difference method (for 2D and $3 \mathrm{D}$ rigid particles) and the finite element method (for 3D capsule) are utilized to solve the dynamics of solid structures. The present method and structure models are validated by several cases: 2D OldroydB channel flow, 2D lid-driven cavity flow, 2D Oldroyd-B flow over a confined cylinder, a 2D rigid 
(a)

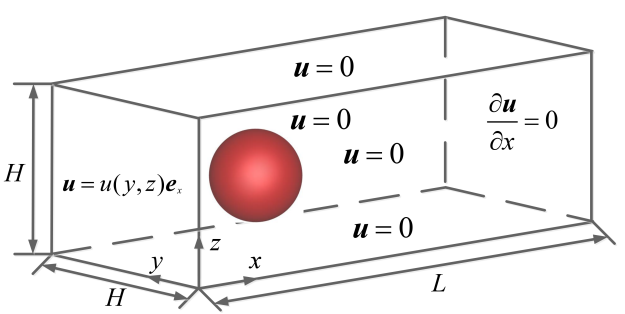

(b)

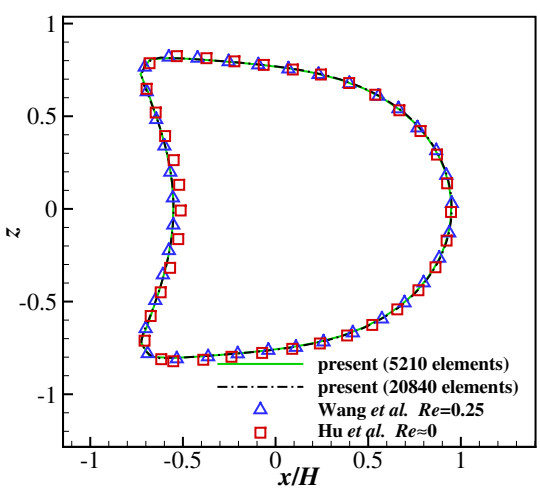

Figure 8: A 3D spherical capsule deformation in a channel with the square cross-section: (a) The Schematic figure for a 3D spherical capsule deformation in a Newtonian channel flow. (b) Steady profiles of an initially spherical capsule with an SK membrane $(C=1)$ flowing in a square section channel for $2 R=0.85 H, R e=0.25$, and $C a=0.3$.

particle migration in an Oldroyd-B Couette flow, a spherical particle rotation in an Oldroyd-B shear flow, a spherical particle settling in a Newtonian fluid, the deformation of a spherical capsule in a long channel filled with a Newtonian fluid. The numerical results are successfully validated against previous experimental and numerical results, and the accuracy of the present method is confirmed.

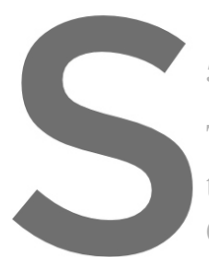

5 ACKNOWLEDGEMENT This research is undertaken with ture, which is supported by th Council Discovery Early
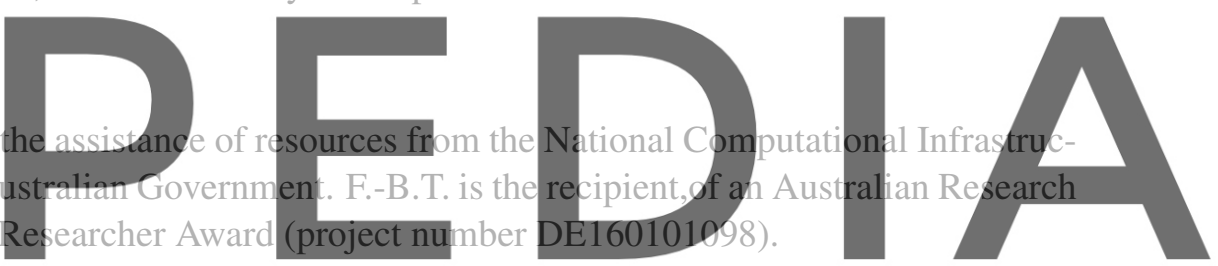

Register for free at https//www.scipedia.com to download the version without the watermark

[1] GJ Li, A Karimi, and AM Ardekani. Effect of solid boundaries on swimming dynamics of microorganisms in a viscoelastic fluid. Rheologica Acta, 53:911-926, 2014.

[2] B Liu, TR Powers, and KS Breuer. Force-free swimming of a model helical flagellum in viscoelastic fluids. PNAS, 108:19516-19520, 2011.

[3] FB Tian. Deformation of a Capsule in a Power-Law Shear Flow. Computational and Mathematical Methods in Medicine, 2016:7981386, 2016.

[4] FB Tian, and L Wang. Numerical modeling of sperm swimming. Fluids, 6:73, 2021.

[5] J Ma, FB Tian, J Young, and JCS Lai. Dynamic behaviours of a filament in a viscoelastic uniform flow. Fluids, 6:90, 2021.

[6] J Feng, PY Huang, and DD Joseph. Dynamic simulation of sedimentation of solid particles in an Oldroyd-B fluid. Journal of Non-Newtonian Fluid Mechanics, 63:63-88, 1996.

[7] MM Villone, F_ Greco, MA Hulsen, and PL Maffettone. Simulations of an elastic particle in Newtonian and viscoelastic fluids subjected to confined shear flow. Journal of Non-Newtonian 
Fluid Mechanics, 210:47-55, 2014.

[8] TE Tezduyar, M Behr, and J Liou. A new strategy for finite element computations involving moving boundaries and interfaces - the deforming-spatial-domain/space-time procedure: I. The concept and the preliminary numerical tests. Computer Methods in Applied Mechanics and Engineering, 94:339 - 351, 1992.

[9] FB Tian, R P Bharti, and YQ Xu. Deforming-spatial-domain/stabilized space-time (DSD/SST) method in computation of non-Newtonian fluid flow and heat transfer with moving boundaries. Computational Mechanics, 53:257-271, 2014.

[10] FB Tian. FSI modeling with the DSD/SST method for the fluid and finite difference method for the structure. Computational Mechanics, 54:581-589, 2014.

[11] FB Tian, H Luo, L Zhu, JC Liao, and XY Lu. An efficient immersed boundary-lattice Boltzmann method for the hydrodynamic interaction of elastic filaments. Journal of Computational Physics, 230:7266-7283, 2011.

[12] FB Tian, H Dai, H Luo, JF Doyle, and B Rousseau. Fluid-structure interaction involving large deformations: 3D simulations and applications to biological systems. Journal of Computational Physics, 258:451-469, 2014.

[13] WX Huang, and FB Tian. Recent trends and progresses in the immersed boundary method. Journal of Mechanical Engineering Science, 233:7617-7636, 2019.

[14] N Goyal, and JJ Derksen. Direct simulations of spherical particles sedimenting in viscoelastic fluids. Journal of Non-Newtonian Fluid Mechanics, 183:1-13, 2012.

[15] L Zhu. A three-dimensional immersed boundary method for non-Newtonian fluids. Theoretical and Applied Mechanics Letters, 8:193-196, 2018.

[16] J Ma, Z Wang, J Young, JCS Lai, Y Sui, and FB Tian. An immersed boundary-lattice Boltzmann method for fluid-structure interaction problems involving viscoelastic fluids and complex geometries. Journal of Computational Physics, 415:109487, 2020.

[17] L Zhu. An IB method for non-Newtonian-fluid flexible-structure interactions in three-dimensions. Computer Modeling in Engineering \& Sciences, 119:125-143, 2019.

[18] O Malaspinas, N Fiétier, and M Deville. Lattice Boltzmann method for the simulation of viscoelastic fluid flows. Journal of Non-Newtonian Fluid Mechanics, 165:1637-1653, 2010.

[19] YK Lee, and KH Ahn. A novel lattice Boltzmann method for the dynamics of rigid particles suspended in a viscoelastic medium. Journal of Non-Newtonian Fluid Mechanics, 244:75-84, 2017.

[20] Z Wang, Y Sui, A-V Salsac, D Barthès-Biesel, and W Wang. Path selection of a spherical capsule in a microfluidic branched channel: towards the design of an enrichment device. Journal of Fluid Mechanics, 849:136-162, 2018.

[21] XQ Hu, B Sévénié, AV Salsac, E Leclerc, and D Barthès-Biesel. Characterizing the membrane properties of capsules flowing in a square-section microfluidic channel: Effects of the membrane constitutive law. Physical Review E, 87:063008, 2013. 
[22] W Helfrich. Elastic properties of lipid bilayers: theory and possible experiments. Zeitschrift für Naturforschung C, 28:693-703, 1973.

[23] ZC Ou-Yang and W Helfrich. Bending energy of vesicle membranes: General expressions for the first, second, and third variation of the shape energy and applications to spheres and cylinders. Physical Review A, 39:5280, 1989.

[24] L Zhu, X Yu, N Liu, Y Cheng, and X Lu. A deformable plate interacting with a non-Newtonian fluid in three dimensions. Physics of Fluids, 29:083101, 2017.

[25] YQ Xu, FB Tian, HJ Li, and YL Deng. Red blood cell partitioning and blood flux redistribution in microvascular bifurcation. Theoretical and Applied Mechanics Letters, 2:024001, 2012.

[26] YQ Xu, XY Tang, FB Tian, YH Peng, Y Xu, and YJ Zeng. IB-LBM simulation of the haemocyte dynamics in a stenotic capillary. Computer Methods in Biomechanics and Biomedical Engineering, 17:978-985, 2014.

[27] Z Liu, FB Tian, J Young, and JCS Lai. Flapping foil power generator performance enhanced with a spring-connected tail. Physics of Fluids, 29:123601, 2017.

[28] J Ma, L Xu, FB Tian, J Young, and JCS Lai. Dynamic characteristics of a deformable capsule in a simple shear flow. Physical Review E, 99:023101, 2019.

[29] S Dalal, G Tomar, and P Dutta. Numerical study of driven flows of shear thinning viscoelastic fluids in rectangular cavities. Journal of Non-Newtonian Fluid Mechanics, 229:59-78, 2016.

[30] S Krishnan, ESG Shaqfeh, and G Iaccarino. Fully resolved viscoelastic particulate simulations using unstructured grids. Journal of Computational Physics, 338:313-338, 2017.

[31] MA Alves, FT Pinho, and PJ Oliveira. The flow of viscoelastic fluids past a cylinder: finite-volume high-resolution methods. Journal of Non-Newtonian Fluid Mechanics, 97:207-232, 2001.

[32] PY Huang, J Feng, HH Hu, and DD Joseph. Direct simulation of the motion of solid particles in Couette and Poiseuille flows of viscoelastic fluids. Journal of Fluid Mechanics, 343:73-94, 1997.

[33] H Lv, SL Tang, and WP Zhou. Direct numerical simulation of particle migration in a simple shear flow. Chinese Physics Letters, 28:084708, 2011.

[34] F Snijkers, G D’Avino, PL Maffettone, F Greco, MA Hulsen, and J Vermant. Effect of viscoelasticity on the rotation of a sphere in shear flow. Journal of Non-Newtonian Fluid Mechanics, 166:363$372,2011$.

[35] A Miyamura, S Iwasaki, and Ta Ishii. Experimental wall correction factors of single solid spheres in triangular and square cylinders, and parallel plates. International Journal of Multiphase Flow, 7:41-46, 1981.

[36] CK Aidun, Y Lu, and EJ Ding. Direct analysis of particulate suspensions with inertia using the discrete Boltzmann equation. Journal of Fluid Mechanics, 373:287-311, 1998.

[37] XQ Hu, A-V Salsac, and D Barthès-Biesel. Flow of a spherical capsule in a pore with circular or square cross-section. Journal of Fluid Mechanics, 705:176-194, 2012. 